\title{
Diffusion with a Discontinuous Potential: a Non-Linear Semigroup Approach
}

\author{
Yong-Jung $\mathrm{Kim}^{1, *}$ and Marshall Slemrod ${ }^{2}$ \\ ${ }^{1}$ Department of Mathematical Sciences, KAIST, 291 Daehak-ro, Yuseong-gu, \\ Daejeon 305-701, Korea \\ ${ }^{2}$ Department of Mathematics, University of Wisconsin, Madison WI 53706, USA
}

Received 31 May 2020; Accepted (in revised version) 23 July 2020

Dedicated to Prof. Paul H. Rabinowitz with admiration on the occasion of his 80th birthday

\begin{abstract}
This paper studies existence of mild solution to a sharp cut off model for contact driven tumor growth. Analysis is based on application of the Crandall-Liggett theorem for $\omega$-quasi-contractive semigroups on the Banach space $L^{1}(\Omega)$. Furthermore, numerical computations are provided which compare the sharp cut off model with the tumor growth model of Perthame, Quirós, and Vázquez [13].
\end{abstract}

Key Words: Nonlinear semigroups, tumor growth models, Hele-Shaw diffusion.

AMS Subject Classifications: 35Q92, 92C50

\section{Introduction}

In their paper [13], Perthame, Quirós, and Vázquez proposed the following model for tumor growth

$$
v_{t}=\nabla \cdot(v \nabla p)+v(1-p), \quad v(0)=v_{0},
$$

where $v$ is the cell density, $v_{0}$ is the initial value, and $p$ is the pressure field. In their model, the pressure field is approximated by

$$
p \cong p_{m}:=\frac{m}{1-m}\left(\frac{v}{v_{c}}\right)^{m-1},
$$

where the coefficient $v_{\mathcal{c}}$ is the maximum packing density and is set to $v_{c}=1$ for convenience. In this case, Eq. (1.1) is written as

$$
v_{t}=\Delta v^{m}+v\left(1-p_{m}\right)
$$

${ }^{*}$ Corresponding author. Email addresses: yongkim@kaist. edu (Y-J. Kim), slemrod@math . wisc.edu (M. Slemrod) 
The contact driven tumor growth model is taken as limit $m \rightarrow \infty$ of (1.3). Perthame, Quirós, and Vázquez [13] proved that, if the initial value is smooth and bounded

$$
0 \leq v_{0} \leq 1,
$$

the pair $\left(v_{m}, p_{m}\right)$ converges to $\left(v_{\infty}, p_{\infty}\right)$ as $m \rightarrow \infty$ which satisfy a Hele-Shaw type diffusion model

$$
\partial_{t} v_{\infty}=\Delta p_{\infty}+v_{\infty}\left(1-p_{\infty}\right), \quad v_{\infty}(0)=v_{0},
$$

in the sense of distributions. Furthermore,

$$
v_{\infty} \in C\left((0, \infty) ; L^{1}\left(\mathbb{R}^{N}\right) \cap B V\left(\mathbb{R}^{N} \times(0, \infty)\right), \quad 0 \leq v_{\infty} \leq 1,\right.
$$

and

$$
p_{\infty} \in P_{\infty}\left(v_{\infty}\right), \quad 0 \leq p_{\infty} \leq 1,
$$

where the inclusion relation (1.6) is given by the set-valued function

$$
P_{\infty}(v)= \begin{cases}0, & 0 \leq v<1 \\ {[0, \infty),} & v=1\end{cases}
$$

Note that the diffusion term in (1.5) is present only when $v_{\infty}=1$. Indeed, the limiting case gives an extreme scenario that the domain is divided into two parts, specifically when (a) the diffusion does not appear at all or (b) it is concentrated at $v=1$.

We note the Hele-Shaw diffusion equation, (1.5)-(1.7), cannot be used as a model for the limiting case. First, it does not single out a solution (though to be fair the extended version of (3.1a) that we introduce in Section 3 will also be defined by an inclusion relation). The main reason is that the set-valued function $P_{\infty}(v)$ has discontinuity at the stable steady state of the reaction term, $v=1$. Furthermore, if the initial value is not bounded by (1.4), the solution is not defined. As an alternative system, we consider a sharp cut off model

$$
u_{t}=\Delta G(u)+f(u), \quad u(0)=u_{0} \geq 0,
$$

where

$$
\begin{aligned}
& G(u)= \begin{cases}0, & u<1, \\
1, & u \geq 1,\end{cases} \\
& f(u)= \begin{cases}u, & 0 \leq u<1, \\
0, & u \geq 1,\end{cases}
\end{aligned}
$$

which has been introduced by Kim and Pan [11]. We set $G(1)=1$ in (1.9a) to connect the model to the nonlinear diffusion in (1.3) which has the same property, i.e., $v^{m}=1$ when $v=1$. The value of the potential at the discontinuity point $u=1$ makes a difference since it is a stable steady state of the reaction function $f(u)$. 
Kim and Pan suggested that the cut off model (1.8)-(1.9b) provides an alternative to (1.3)-(1.4) with $m \gg 1$ large. To check their conjecture Kim and Pan performed numerical experiments and their results are here illustrated in Fig. 1 of Section 4 . We observe that that numerical results for (1.3) with $m$ large and (1.8)-(1.9b) are almost identical.

The apparent success of the Kim-Pan model of course raises the mathematical issue as to existence of solutions to (1.8)-(1.9b) for appropriately given initial and boundary conditions. To address this issue we will place the problem within the context of nonlinear semigroups of $\omega$-quasi-contractions on the Banach space $L^{1}(\Omega)$. The advantage of this formulation is obvious: we will only need use of the existing mathematical theory as provided by the classical Crandall-Liggett theorem [6].

This paper has three sections after this Introduction. Section 2 provides a review of the theory of $m$-accretive operators and non-linear semigroup theory on Banach spaces. Section 3 applies this theory to obtain the existence of mild solution to system (1.8)-(1.9b). Section 4 gives careful comparisons of numerical solutions of (1.3) and (1.8)-(1.9b). In particular, we observed there is nice convergence solutions of (1.3) to a solution of (1.8)$(1.9 b)$ when the CFL condition is satisfied. However, when the CFL condition is violated, solutions of (1.3) blow up where as solutions of (1.8)-(1.9b) remain bounded albeit with oscillations.

\section{Review of $m$-accretive operators and non-linear semigroups}

We follow the presentations of Evans [9] and Barbu [1] though the definitions are standard (also see $[2,12]$ ). Let $\mathbb{X}$ be a Banach space with norm $\|\cdot\|$. An operator $A: D(A) \rightarrow$ $\mathbb{X}$ with its domain $D(A) \subset \mathbb{X}$ is called accretive if

$$
\|u-v\| \leq\|u-v+\lambda(A(u)-A(v))\|
$$

for all $u, v \in D(A)$ and $\lambda \in \mathbb{R}_{+}$. If, in addition, Range $(I+\lambda A)=\mathbb{X}$ for some (equivalently for all) $\lambda>0$, then $A$ is called $m$-accretive. A simple way to check accretiveness in examples is to define

$$
[u, v]_{+}=\inf _{\lambda>0} \frac{\|u+\lambda v\|-\|u\|}{\lambda}
$$

Then the operator $A$ is accretive if and only if

$$
0 \leq[u-v, A(u)-A(v)]_{+} \text {for all } u, v \in D(A) .
$$

When $\mathbb{X}=L^{1}(\Omega)$ for a bounded domain $\Omega \subset \mathbb{R}^{n}$, we may use the result of Sato [14]:

$$
[u, v]_{+}=\int_{u>0} v d x-\int_{u<0} v d x+\int_{u=0}|v| d x .
$$


We are interested in resolving the initial value problem

$$
\begin{aligned}
& u_{t}+A u \ni 0, \quad 0<t<\infty, \\
& u(0)=u_{0} \in D(A),
\end{aligned}
$$

where $A$ is an $m$-accretive set-valued operator.

In their classic paper, Crandall and Liggett [6] provided a mild solution to (2.4a), $(2.4 \mathrm{~b})$ via a sequence of discrete problems where the time derivative in (2.4a) is replaced by a difference quotient:

$$
\frac{u(t)-u(t-\varepsilon)}{\varepsilon}+A u(t) \ni 0
$$

for $\varepsilon>0$ small so that $(2.4 \mathrm{~b}),(2.5)$ can be solved recursively. We summarize their results as follows.

If $C \subset \mathbb{X}$, a semigroup on $C$ is a function $S$ on $[0, \infty)$ such that $S(t)$ maps $C$ into $C$ for each $t \geq 0$ and satisfies

$$
\begin{array}{ll}
S(t+\tau)=S(t) S(\tau) & \text { for } t, \tau \geq 0, \\
\lim _{t \rightarrow 0^{+}} S(t) u=S(0) u=u & \text { for } u \in C .
\end{array}
$$

If $S$ is a semigroup on $C$ and there is a real number $\omega$ so that

$$
\|S(t) u-S(t) v\| \leq e^{\omega t}\|u-v\|
$$

for $t \geq 0$ and $u, v \in C$, we say the semigroup is $\omega$-quasi-contractive.

Theorem 2.1 (Crandall-Liggett [6]). Let $A$ be a possibly set-valued operator $A: D(A) \rightarrow \mathbb{X}$ such that there is a real number $\omega$ with $A+\omega I$ being accretive. If $\overline{D(A)} \subset R(I+\lambda A)$ for all sufficiently small $\lambda>0$, then

$$
\lim _{n \rightarrow \infty}\left(I+\frac{t}{n} A\right)^{n} u_{0}
$$

exists for all $u_{0} \in \overline{D(A)}$ and $t>0$. Moreover, if $S(t) u_{0}$ is defined as this limit in $C$, then $S$ is a $\omega$-quasi-contractive semigroup on $\overline{D(A)}$.

We call $u(t):=S(t) u_{0}$ a mild solution of (2.4a), (2.4b).

In general, we do not know that $D(A)$ is invariant under the map $S(t)$ (unlike the case of linear semigroups where $u(t)=S(t) u_{0}, u_{0} \in D(A)$, provides a strong solution of (2.4a), (2.4b), see e.g., [10]). However, there is a generalized domain $\hat{D}(A)$ defined by Crandall [7], which is invariant under $S(t)$. In particular, $S(t) u_{0}$ is locally Lipschitz continuous in $t, u_{0} \in \hat{D}(A)$.

In the initial-boundary value problem of Section 3, we will be interested in the case of $\mathbb{X}=L^{1}(\Omega)$ and thus the nonlinear semigroup theory for reflexive Banach spaces does not apply (see for example Barbu [1], Evans [8,9], and Zeidler [15]). 


\section{Existence}

We consider the initial-boundary value problem

$$
\begin{array}{ll}
u_{t}=\Delta G(u)+f(u) & \text { in } \Omega \subset \mathbb{R}^{N}, t>0, \\
u=0 & \text { on } \partial \Omega, \\
u=u_{0} \in L^{1}(\Omega) & \text { at } t=0,
\end{array}
$$

where $\Omega$ is a bounded open set in $\mathbb{R}^{N}$ with a smooth boundary. Here,

$$
\begin{aligned}
& G(u)= \begin{cases}0, & u<1, \\
1, & u \geq 1,\end{cases} \\
& f(u)= \begin{cases}0, & u<0, \\
u, & 0 \leq u<1, \\
0, & u \geq 1 .\end{cases}
\end{aligned}
$$

Note

$$
q(u):=f(u)-u= \begin{cases}-u, & u<0 \\ 0, & 0 \leq u<1 \\ -u, & u \geq 1\end{cases}
$$

and hence $-g(u)=u-f(u)$ has a monotone increasing graph. Next, note

$$
g(u):=q(u)+G(u)= \begin{cases}-u, & u<0 \\ 0, & 0 \leq u<1 \\ -u+1, & u \geq 1\end{cases}
$$

and hence $-g(u)$ is continuous on $\mathbb{R}$ with a monotone graph. Thus

$$
\begin{aligned}
& -g(u)=-q(u)-G(u)=-f(u)+u-G(u), \\
& f(u)=g(u)+u-G(u),
\end{aligned}
$$

and we can rewrite (2.1), (2.2) as

$$
\begin{array}{ll}
u_{t}=(\Delta-I) G(u)+g(u)+u & \text { in } \Omega \subset \mathbb{R}^{N}, t>0, \\
u=u_{0} \in L^{1}(\Omega) & \text { at } t=0 .
\end{array}
$$

Next, we recall two results given by Brézis-Strauss [3] and Barbu [1].

Proposition 3.1. Let $\mathbb{X}$ be a real Banach space, $A$ an m-accretive operator, and $B$ a continuous m-accretive operator with $D(B)=\mathbb{X}$. Then, $A+B$ is m-accretive. 
Proposition 3.2 (Barbu [1], p. 114). Let $\mathbb{X}=L^{1}(\Omega)$. Define the operator

$$
\begin{aligned}
& A u:=-\Delta \beta(u) \quad \text { for } u \in D(A), \\
& D(A)=\left\{u \in L^{1}(\Omega) ; \beta(u) \in W_{0}^{1,1}(\Omega), \Delta \beta(u) \in L^{1}(\Omega)\right\},
\end{aligned}
$$

where $\beta$ is a maximum monotone graph in $\mathbb{R} \times \mathbb{R}$ with $0 \in \beta(0)$ and $\Omega$ is an open bounded subset of $\mathbb{R}^{N}$ with smooth boundary. Then, the operator $A$ is m-accretive in $L^{1}(\Omega) \times L^{1}(\Omega)$.

We note Remark 3.1 (Barbu [1, p. 114]) that $\Delta$ can be replaced by any second order elliptic linear operator on $\Omega$. In other words, Proposition 3.2 applies to the operator $(-\Delta+I) \bar{G}(u)$ since $(a)(-\Delta+I)$ is a second order linear elliptic operator and $(b) \bar{G}$ has a maximal monotone graph where

$$
\bar{G}= \begin{cases}0, & u<1 \\ {[0,1],} & u=1 \\ 1, & u>1\end{cases}
$$

Lemma 3.1. The map $-g: \mathbb{R} \rightarrow \mathbb{R}$ is continuous and m-accretive on $L^{1}(\Omega)$.

Proof. The map $-g: \mathbb{R} \rightarrow \mathbb{R}$ is globally Lipschitz continuous: $|g(u)-g(v)| \leq L|u-v|$. This implies

$$
\|g(u)-g(v)\|_{L^{1}(\Omega)} \leq L\|u-v\|_{L^{1}(\Omega)} .
$$

Furthermore, Sato's lemma of Section 2 implies $-g: L^{1}(\Omega) \rightarrow L^{1}(\Omega)$ is accretive. Finally, the range condition $u+\lambda g(u)=f, f \in L^{1}(\Omega)$ is satisfied by solving this equation for each $u$ :

$$
u= \begin{cases}(1+\lambda)^{-1} f, & f<0, \\ f, & 0 \leq f \leq 1, \\ (1+\lambda)^{-1} f+1, & f>1 .\end{cases}
$$

Clearly, $f \in L^{1}(\Omega)$ implies $u \in L^{1}(\Omega)$.

Lemma 3.2. The operator $A_{1}$ defined by

$$
A_{1} u:=(-\Delta+I) \bar{G}(u)-g(u)
$$

is m-accretive on $L^{1}(\Omega)$ where

$$
D\left(A_{1}\right)=\left\{u \in L^{1}(\Omega) ; \bar{G} \in W_{0}^{1,1}(\Omega), \Delta \bar{G}(u) \in L^{1}(\Omega)\right\} .
$$

Proof. Use Proposition 3.1, Proposition 3.2, and Lemma 3.1.

Lemma 3.3. The operator $A_{2}:=A_{1}-I, D\left(A_{2}\right)=D\left(A_{1}\right)$, satisfies the range condition $R(I+$ $\left.\lambda A_{2}\right)=L^{1}(\Omega)$ for $\lambda>0$, sufficiently small. 
Proof. For $f \in L^{1}(\Omega)$ we wish to solve, $u+\lambda A_{2} u \ni f$, i.e., $(1-\lambda) u+\lambda A_{1} u=f$, or

$$
u+\left(\frac{\lambda}{1-\lambda}\right) A_{1} u \ni \frac{f}{1-\lambda}
$$

Since $A_{1}$ is $m$-accretive, (3.2) possesses a solution $u \in D\left(A_{1}\right)$ for all $f \in L^{1}(\Omega)$.

Finally, the Crandall-Liggett Theorem is applied to show the existence of a solution.

Theorem 3.1. $-A_{2}$ is the generator of a semigroup of $\omega$-quasi-contractions on $\overline{D\left(A_{2}\right)} \subset L^{1}(\Omega)$. For $u_{0} \in \overline{D\left(A_{2}\right)}, u(t)=S(t) u_{0}, t>0$, provides a mild solution of the initial-boundary value problem (3.1a)-(3.1c).

\section{Numerical simulations}

The heat and the Poisson equations are often used as canonical systems to test numerical schemes. In the theory, uniform ellipticity and bounded diffusivity are assumed. However, the diffusion model with a discontinuous potential is an extreme case where both assumptions fail. The behavior of numerical schemes for such discontinuous diffusion models is not usually studied. An explicit finite difference scheme, a forward in time and centered in space scheme (see Appendix), is considered in this section which gives characteristic properties of related numerical schemes in this simple context.

We first test if the numerical solution of

$$
u_{t}=\Delta G(u)+f(u)
$$

gives the same subsequential limit of

$$
v_{t}=\Delta v^{m}+v\left(1-p_{m}\right),
$$

which has been obtained by Perthame et al. [13]. Here, $p_{m}, G$, and $f$ are respectively given by (1.2), (1.9a), and (1.9b). The two model equations are solved numerically and compared in Fig. 1. The computation is done on a domain $\Omega=[-10,10]$ with the zero Dirichlet boundary condition. The initial values are

$$
u_{0}(x)=v_{0}(x)=0.1 \cos (x), \quad \text { if } \quad-\frac{\pi}{2} \leq x \leq \frac{\pi}{2},
$$

and zero otherwise. The solution is symmetric with respect to $x=0$ and hence displayed only on the domain $0<x<10$.

An explicit numerical scheme for a partial differential equation of an advection phenomenon should satisfy the CFL condition, i.e., the Courant number $C$ should be less than one, i.e.,

$$
\frac{s \Delta t}{\Delta x} \leq 1
$$


where $s>0$ is the speed of the advection phenomenon, $\Delta x$ is the space mesh size, and $\Delta t$ is the time step. The Courant number of a numerical scheme for a parabolic problem is given by

$$
C:=\frac{2 d n \Delta t}{|\Delta x|^{2}}
$$

where $d>0$ is the diffusivity and $n \geq 1$ is the space dimension. In the first three numerical computations, we take time and space meshes with

$$
\Delta x=10^{-1}, \quad \Delta t=5 \times 10^{-5} .
$$

The diffusivity of the continuous diffusion model (4.2) is $d=m v^{m-1}$. Hence, if the mesh size is given by (4.4), the Courant number is bounded by

$$
C_{v}:=\frac{m v^{m-1} 2 \Delta t}{|\Delta x|^{2}} \leq m \frac{2 \Delta t}{|\Delta x|^{2}}=m \times 10^{-2},
$$

where the inequality comes from the solution bound $|v| \leq 1$. The Courant number for the discontinuous diffusion model (4.1) is

$$
C_{u}:=G^{\prime}(u) \frac{2 \Delta t}{|\Delta x|^{2}}=G^{\prime}(u) \times 10^{-2},
$$

where $G^{\prime}(u)=0$ for $u \neq 1$ and $G^{\prime}(u)=\infty$ for $u=1$. The CFL stability condition is " $C \leq 1$ ", which is a necessary condition for the stability of a numerical scheme. Hence, if the discretization is given by (4.4), the numerical solution of (4.2) satisfies the CFL condition if $m \leq 100$. We indeed observe that numerical solutions blow up soon after $m>100$. On the other hand, since the discontinuous diffusion model (4.1) is the limiting case of (4.2) as $m \rightarrow \infty$, one may expect that its numerical solution blows up as well. However, we found that the numerical scheme is actually stable and stays bounded as long as $\frac{2 \Delta t}{|\Delta x|^{2}}<1$, which is the CFL condition for the constant diffusivity case with $d=1$. This observation is quite surprising and requires a better understanding.

In Fig. 1, snap shots of numerical solutions of the two models are given at $t=10$. See Appendix ?? for the matlab code of this computation. Solutions of (4.2) clearly converge to the solution of the cut off model (4.1) as $m \rightarrow \infty$. This convergence is monotone and convinces us that the solution of the cut off model (4.1) is the limit of Perthame et al. [13] that satisfies the Hele-Shaw diffusion equation, (1.5)-(1.7).

In Fig. 2, the cell density and the diffusion pressure for the two models are compared when $m=100$. The figure in the right shows that the diffusion pressure $p_{m}$ of the continuous model (4.2) connects the interface and inside cells monotonically. This profile is consistent in time and propagates with the front without changing its shape. On the other hand, the potential $G$ of the discontinuous model (4.1), which also plays the role of the pressure, oscillates as in the figure in the left. The position and size of the oscillating region varies as the solution propagates. However, the inconsistent behavior is completely averaged out and the cell growth interfaces of the two models agree perfectly. 

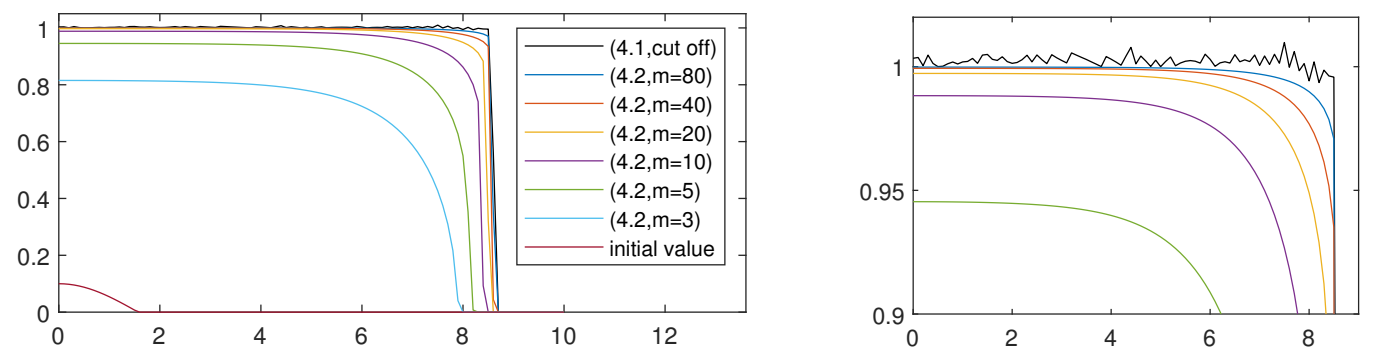

Figure 1: Snap shots of (4.1) and (4.2) at $t=10$. Mesh sizes are $\Delta x=0.1$ and $\Delta t=5 \times 10^{-5}$.
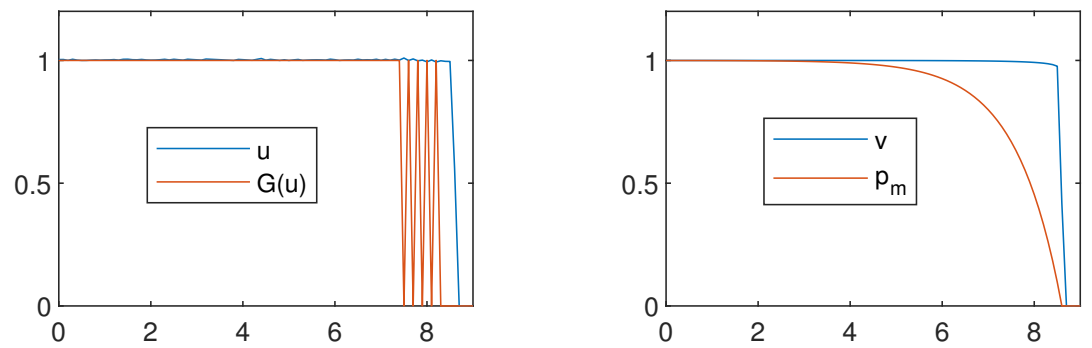

Figure 2: Snap shots of cell density and potential for (4.1) and (4.2). We took $m=100$ and $t=10$.

In Fig. 3, we observe numerically what happens when the CFL condition fails. The Courant number for the numerical solution of the continuous diffusion model (4.2) is $C_{v}=m \times 10^{-2}$ when the mesh is given by (4.4). Hence, the CFL condition fails if $m>$ 100 , which is why we did the computation for $m \leq 100$ in Fig. 1. Indeed, if $m=102$, the numerical solution blows up and becomes unbounded in a finite time. In the right of Fig. 1, the numerical solutions are magnified for values between 0.9 and 1.2. One can see that the numerical solution of the discontinuous cut off model oscillates. This is because of the discontinuity of the diffusion potential $G$ and the fact that $u=1$ is a stable steady state. Note that even a small numerical error near the steady state $u=1$ gives large oscillating noise in $\Delta G(u)$ due to the discontinuity of the potential $G$ and produces the oscillation. We can also see that the solution of the cut off model (4.1) stays above other solutions. To see this more clearly, the solutions are magnified near the steady state $u=1$ in Fig. 3. See the figure in the left and find that numerical solutions for the nonlinear diffusion model (4.2) increase as $m$ increases and stay below the solution of the sharp cut off model (4.1). However, even the solution of the nonlinear diffusion model oscillates when $m=101$, i.e., when the CFL condition fails (see the figure in the right). The solution of the discontinuous model (4.1) is not an upper bound of the solution of the continuous model (4.2) anymore. If $m=102$, the solution blows up entirely and becomes unbounded.

In Fig. 4, three snap shots of the numerical solution of the cut off model (4.1) are given 

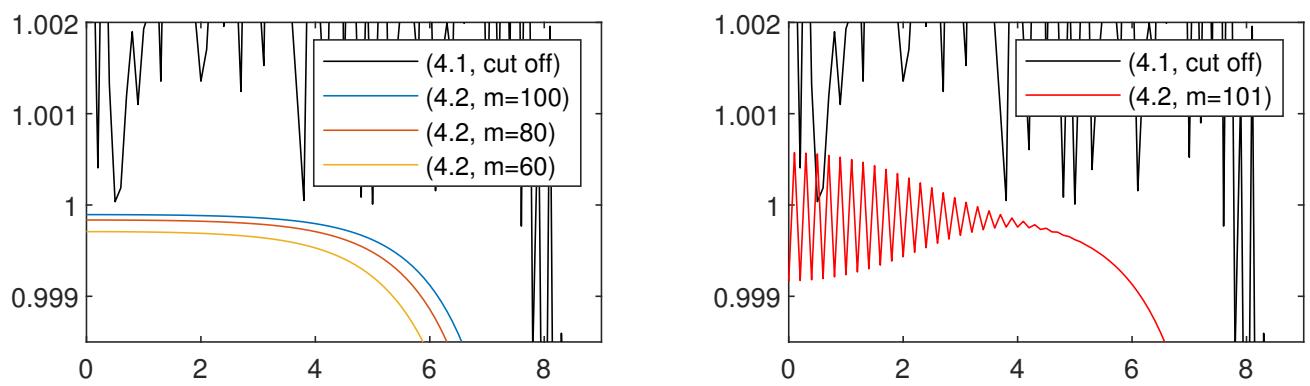

Figure 3: Magnified snap shots of (4.1) and (4.2) at $t=10$.
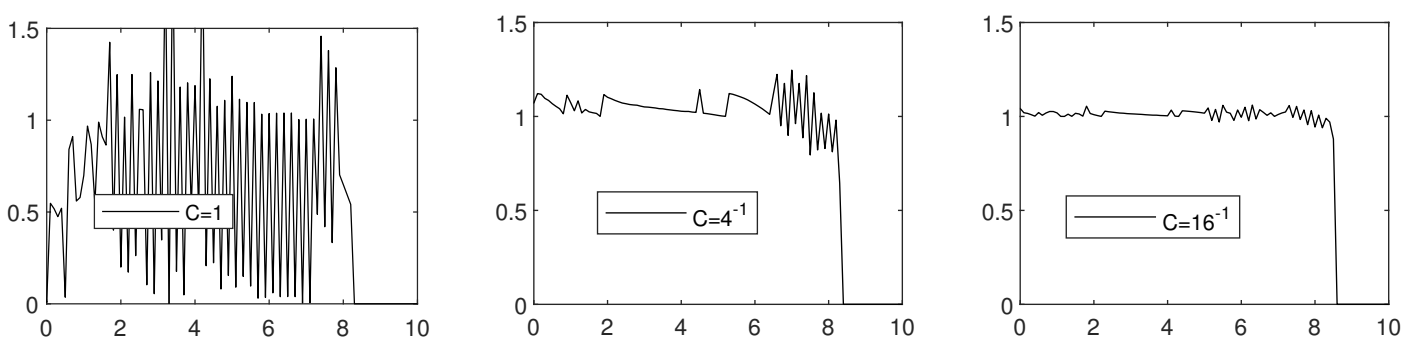

Figure 4: Snap shots of (4.1) at $t=10$. We took $\Delta x=0.1$ fixed and $\Delta t=0.005,0.0013$, and 0.00033 from left.

with different Courant numbers. The space mesh size is taken with $\Delta x=0.1$ and three different time mesh sizes taken with

$$
\Delta t=0.005,0.0013, \text { and } 0.00033 \text {. }
$$

Notice that the Courant number $C_{u}$ in (4.5) is not defined since $G^{\prime}(u)=\infty$ when $u=1$. The Courant number denoted in Fig. 4 is the one for the constant diffusivity case given in (4.3) with $d=1$ and $n=1$. We observe that the solution oscillates with any Courant number. However, the solution is numerically stable as long as the Courant number is less than one, i.e., if the CFL condition for a constant diffusivity case is satisfied. If $C>1$, both numerical solutions of the discontinuous diffusion model (4.1) and of the constant diffusivity one blow up together. It is unexpected that the discontinuous diffusion model (4.1) is more stable than the continuous nonlinear diffusion model (4.2).

In Fig. 5, we observe that the blowup behavior of the continuous diffusion model (4.2) is consistent. We take $\Delta x=0.1$ fixed and three cases of

$$
\Delta t=0.0013,0.00065 \text {, and } 0.00033 \text {. }
$$

In these three cases, the Courant numbers of the unit diffusivity cases are respectively $C=4^{-1}, 8^{-1}$, and $16^{-1}$. Numerical solutions of the cut off model (4.1) are denoted by $u$ in the figures. The numerical solutions of the continuous model (4.2) are given with borderline exponents $m$ which makes a solution about to blow up. We may observe that these $m \times C \gtrsim 1$, i.e., $C_{v} \gtrsim 1$. 

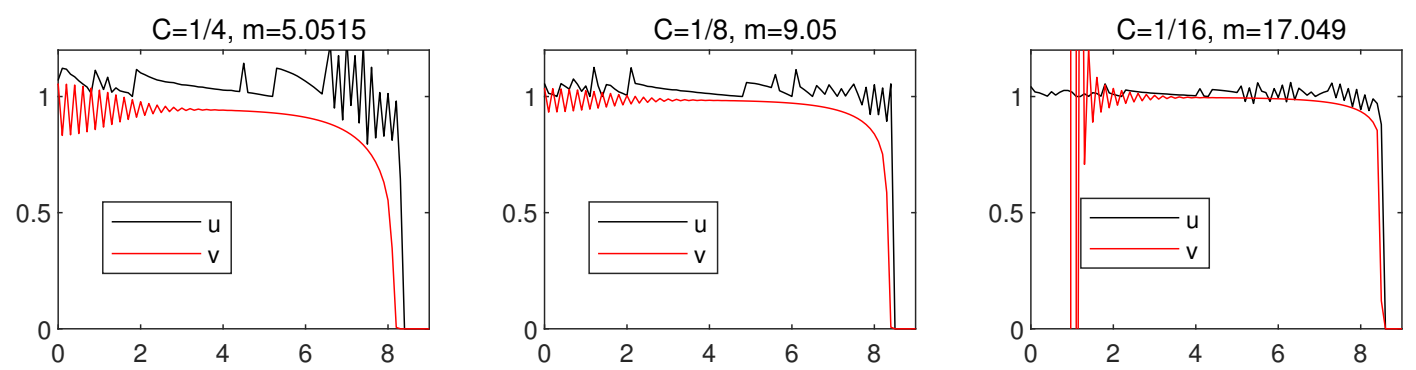

Figure 5: Snap shots of (4.1) and (4.2) at $t=10 . \Delta x=0.1$ and $\Delta t=0.0013,0.00065$ and 0.00033 from left.

\section{Conclusions}

The diffusion equation (1.8) with a discontinuous diffusion potential $G$ can be used as a simplified model for contact driven tumor growth [13], finite time extinction [5], obstacle problems [4], and etc. However, since most theories of parabolic and elliptic problems are based on bounded diffusivity, such equations are rarely studied. In this paper we demonstrated that nonlinear semigroup theory is applicable to such extreme cases and obtained the existence of a mild solution. We also found that a numerical scheme applied to a discontinuous diffusion model (4.1) is more stable than expected. It surprisingly gives the correct interface of tumor growth even when the numerical solution for the continuous diffusion model (4.2) blows up.

\section{Appendix: Numerical computation code}

The numerical computations in this paper are based on a matlab code in the below. We have computed the solution changing the parameter $m$ and time step size $d t$, and then displayed them as in figures.

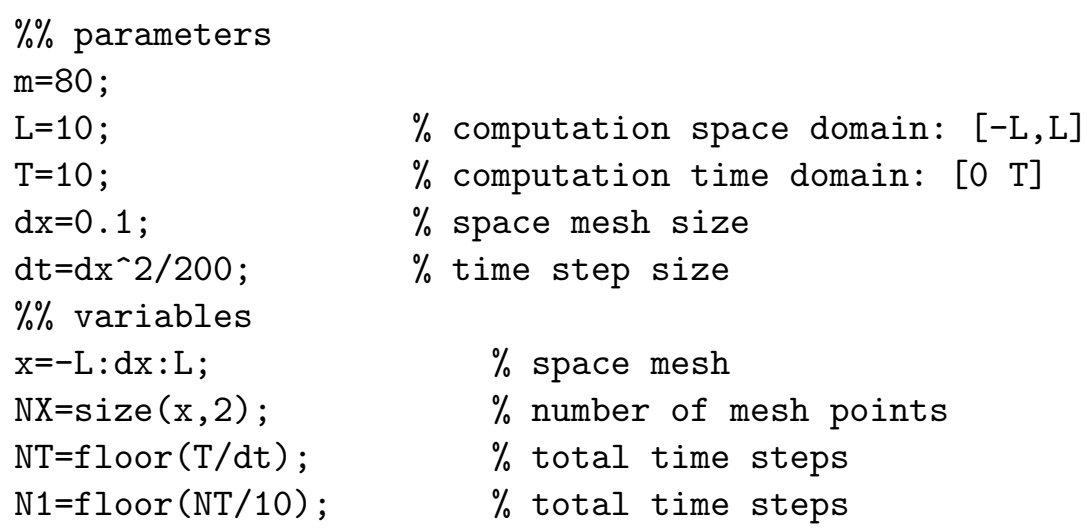




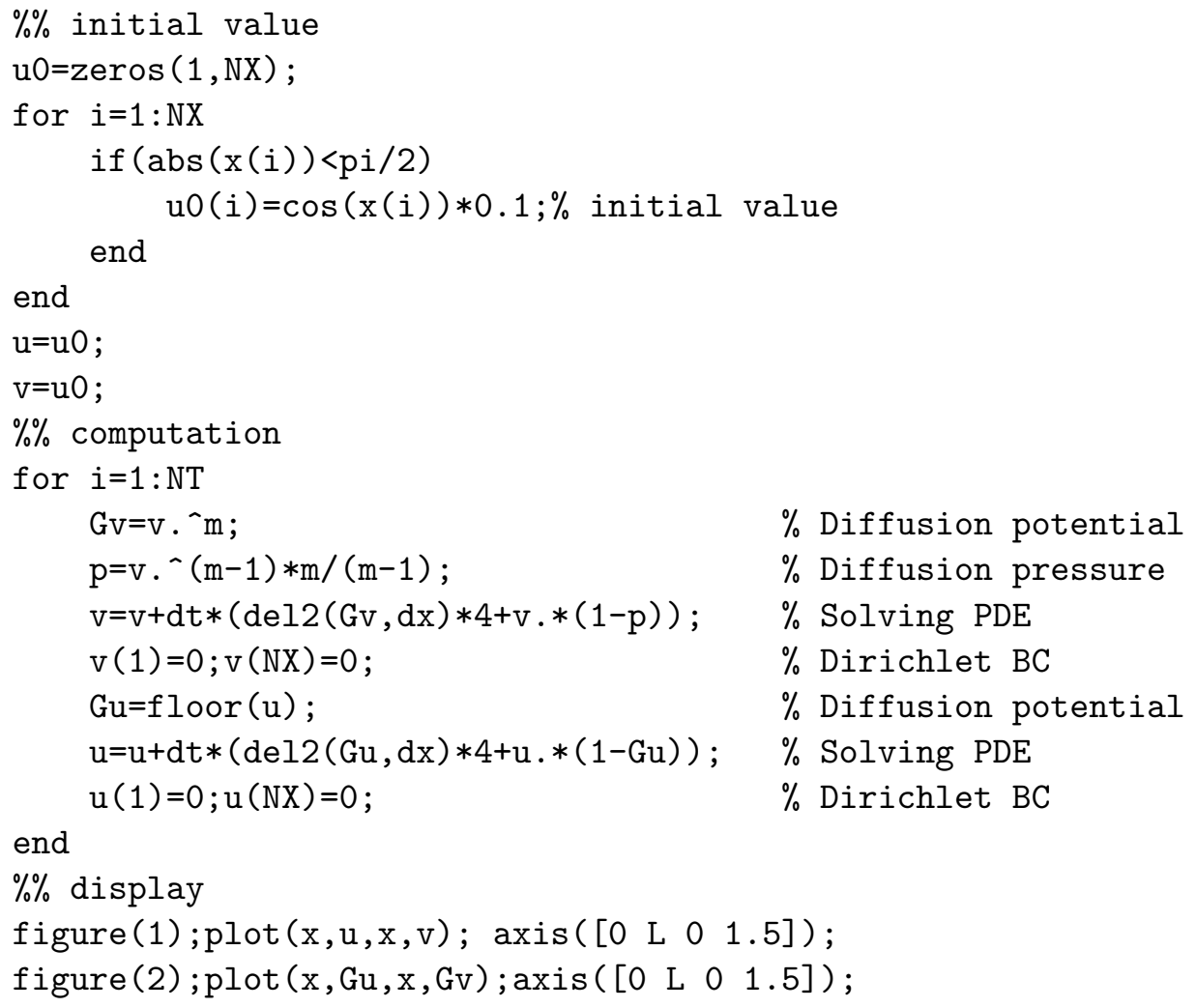

\section{Acknowledgements}

This work was supported in part by National Research Foundation of Korea (NRF2017R1A2B2010398). The authors thank Profs. L. C. Evans and W. Strauss for their valuable suggestions.

\section{References}

[1] V. Barbu, Analysis and Control of Nonlinear Infinite Dimensional Systems, Academic Press, (1993).

[2] P. Bénilan and M. G. Crandall, The continuous dependence on $\varphi$ of solutions of $u_{t}-\Delta \varphi(u)=$ 0, Indiana Univ. Math. J., 30 (1981), 161-177.

[3] H. Brézis and W. A. Strauss, Semi-linear second-order elliptic equations in $L^{1}$, J. Math. Soc. Japan, 25 (1973), 565-590.

[4] X. Chen and C. M. Elliott, Asymptotics for a parabolic double obstacle problem, Proc. Roy. Soc. London Ser. A, 444 (1994), 429-445.

[5] J. Chung, Y.-J. Kim, O. Kwon, and X. Pan, Discontinuous nonlinearity and finite time extinction, SIAM J. Math. Anal., 52 (2020), 894-926.

[6] M. G. Crandall and T. M. Liggett, Generation of semigroups of nonlinear transformations in general Banach spaces, Amer. J. Math., 93 (1971), 265-298. 
[7] M. G. Crandall, A generalized domain for semigroup generators, Proc. Amer. Math. Soc., 37 (1973), 434-440.

[8] L. C. Evans, Nonlinear evolution equations in an arbitrary Banach space, Israel J. Math., 26 (1977), 1-42.

[9] L. C. Evans, Application of nonlinear semigroup theory to vertain partial differential equations, Nonlinear Evolution Equations, Academic Press, (1978), 163-188.

[10] L. C. Evans, Partial differential equations, Graduate Studies in Mathematics, 19. American Mathematical Society, Providence, RI, 1998.

[11] Y.-J. Kim and X. Pan, Coupled solution for discontinuous reaction and diffusion, preprint.

[12] Y. Konishi, On the nonlinear semi-groups associated with $u_{t}=\Delta \beta(u)$ and $\varphi\left(u_{t}\right)=\Delta u$, J. Math. Soc. Japan, 25 (1973), 622-628.

[13] B. Perthame, F. Quiros, and J. L. Vazquez, The Hele-Shaw asymptotics for mechanical models of tumor growth, Arch. Rational Mech. Anal., 212 (2014), 93-127.

[14] K. Sato, On the generation of non-negative contraction semi-groups in Banach lattices, J. Math. Soc. Japan, 20 (1968), 431-436.

[15] E. Zeidler, Nonlinear Functional Analysis and Its Applications III, Variational Methods and Optimization, Springer-Verlag, New York, (1985). 